\title{
A method of laser ultrasound tomography for solid surfaces mapping
}

\author{
Vasily Zarubin ${ }^{1,2, *}$, Anton Bychkov ${ }^{1,2}$, Alexander Karabutov ${ }^{1,3}$, Varvara Simonova ${ }^{4}$ and \\ Elena Cherepetskaya ${ }^{1}$ \\ ${ }^{1}$ National University of Science and Technology "MISiS", 119049 Moscow, Russia \\ ${ }^{2}$ Lomonosov Moscow State University, Faculty of Physics, 119234 Moscow, Russia \\ ${ }^{3}$ Lomonosov Moscow State University, International Laser Centre, 119991 Moscow, Russia \\ ${ }^{4}$ Institute on Laser and Information Technologies of the Russian Academy of Sciences, 140700 \\ Shatura, Moscow Region, Russia
}

\begin{abstract}
Surface control is an important problem for production inspection in industry. Traditionally it is solved using mechanical and optical profilometry, providing high spatial resolution. However, such methods are inappropriate for bodies immersed in opaque liquids and require preliminary preparation of samples. Current work focuses on an application of a method of laser ultrasound tomography to mapping of solid surfaces. The suggested approach combines high acoustical resolution, high performance and allows profilometry of contaminated and immersed samples. An automated prototype for real-time surface profile measurements is constructed and tested on several bodies of revolution. Linear approximation of a cylinder profile obtained by the prototype shows maximum approximation inconsistency of $15 \mu \mathrm{m}$. The results are verified by those obtained by the conventional x-ray method.
\end{abstract}

\section{Introduction}

The problem of solid surface profilometry is important for a wide range of industrial applications. For instance, profilometry is used for an automatic detection of critical damages and defects on the surfaces of pipelines, reservoirs where a high resolution is not required, but the area of controlled surface is large [1]. On the other hand, there are problems requiring high resolution while inspecting small surface areas. These include such tasks as control of polymeric films with complex surface [2], parts for precision systems [3] and many others. In general, profilometry of surface is conducted using methods that can be divided into two groups - contact and non-contact methods.

The most commonly used contact method of profilometry is a mechanical stylus profilometry [4]. Despite the relative simplicity of the device design, the profilers of such type reliably provide a measurement of the solid surfaces with high resolution (up to fractions of nanometres) simultaneously providing a wide range of measurements. Difficulties in inspection of sensitive films and low operation performance are among the

*Corresponding author: zarubin.vasily@gmail.com 
drawbacks of contact profilometers. This often forces technicians to use other methods of profilometry.

The mentioned problems can be solved by using non-contact profilometry methods, such as optical and ultrasonic. The most commonly used methods of optical profilometry are laser interferometry and laser triangulation $[5,6]$. The advantages of these methods include high resolution, comparable with that provided by the stylus profilers, as well as high-speed performance. However, an expensive preliminary cleaning of samples is required for conduction of the optical profilometry. At the same time, industrial and factory control requires the study of contaminated objects, which may be immersed in opaque liquids (for example, oil tanks). In such situations it is convenient to use ultrasonic methods.

Acoustic microscopy is the most appropriate ultrasonic method for measurements of the surface [7]. It uses ultrasound of high frequency greater than $50 \mathrm{MHz}$, which provides a high submicronic longitudinal resolution. However, high frequencies are strongly attenuated in organic liquids, for example, oil, so the characteristic longitudinal resolution of ultrasonic profilometry is of the order of $\sim 10 \mu \mathrm{m}$, which, nevertheless, is sufficient for many industries.

In the present work the laser ultrasound tomography method is used to perform profilometry of objects immersed in liquids. This approach has a number of advantages over traditional methods of profile imaging.

Laser ultrasound is the ultrasound induced by a specific way of generation. The laser beam is delivered to absorbing material, called an optoacoustic generator. Due to absorption in the near-surface layer and subsequent instant heating and short expansion of this substance [8] the ultrasonic waves are excited. The advantage of the laser method of ultrasound generation is the ability to generate powerful aperiodic pulses that are not available to conventional ultrasonic generators. The short submicrosecond duration and smooth shape of the probe pulse leads to an increased longitudinal resolution comparing to the traditional methods of ultrasound microscopy. At the same time, the use of the tomographic approach leads to an increased speed of operation.

\section{Experimental method}

Current work is focused on the development of principles, algorithms and a setup for highspeed laser-ultrasonic profilometry of solids.

The main principle of an experimental method is the following. The radiation from $\mathrm{Nd}$ :YAG laser (wavelength $\lambda=1064 \mathrm{~nm}$, pulse duration $\tau=10 \mathrm{~ns}$, pulse repetition rate $20 \mathrm{~Hz}$, Q-switched) is delivered by an optical fiber (Fig. 1) to the optoacoustic generator for laser excitation of ultrasound. Ultrasonic waves are focused by an acoustic lens on the surface of the object under study, which partially scatters them. The scattered radiation is concentrated by the same lens on the surface of the receiving elements of the acoustic antenna array. The acoustic antenna consists of 16 receiving piezoelectric PVDF transducers, which provide effective reception of a wideband ultrasonic signal in the frequency band of $1.6-9 \mathrm{MHz}$ [9]. Although the transducers are flat, they are glued to the surface of the acoustic lens, what makes the antenna effectively cylindrically focused.

Using a 3-axis positioning system, the sample is moved in the immersion liquid (water) to a focal plane of the acoustic antenna. After that, the sample is irradiated by ultrasound and the scattered signal is recorded by the receiving antenna. Analog electrical signals are fed to the preamplifier, get digitized by a high-speed multi-channel data acquisition and processing system. This system is based on FPGA NI FlexRIO architecture, has a built-in multichannel $\mathrm{ADC}$ with a sampling rate of $50 \mathrm{MHz}$. Its functions are digitizing analog 
signals, storing, averaging and transferring data to a PC for further processing of data and reconstruction of tomograms with a subsequent detection of the surface profile.

To operate the experimental setup a specialized software was developed. This software has a graphical interface that allows to control the sample movement. The software operates in real time (frame rate is $10 \mathrm{~Hz}$ ) and displays tomograms of an object located in the focal plane of the antenna. For the reconstruction of tomogram the filtered back-projection algorithm is used [10]. The algorithm constructs an image over "arcs of probability", which have a shape of parabolas. A plane probing acoustic pulse is scattered by an inhomogeneity and then it's recorded by the receiver elements at a time moment $t=(z+d) / c$, where $z$ is the distance travelled by the probe pulse from the generator to acoustic inhomogeneity, and $d$ is the distance from the inhomogeneity to the receiving transducer.

\section{3-axis positioning system}

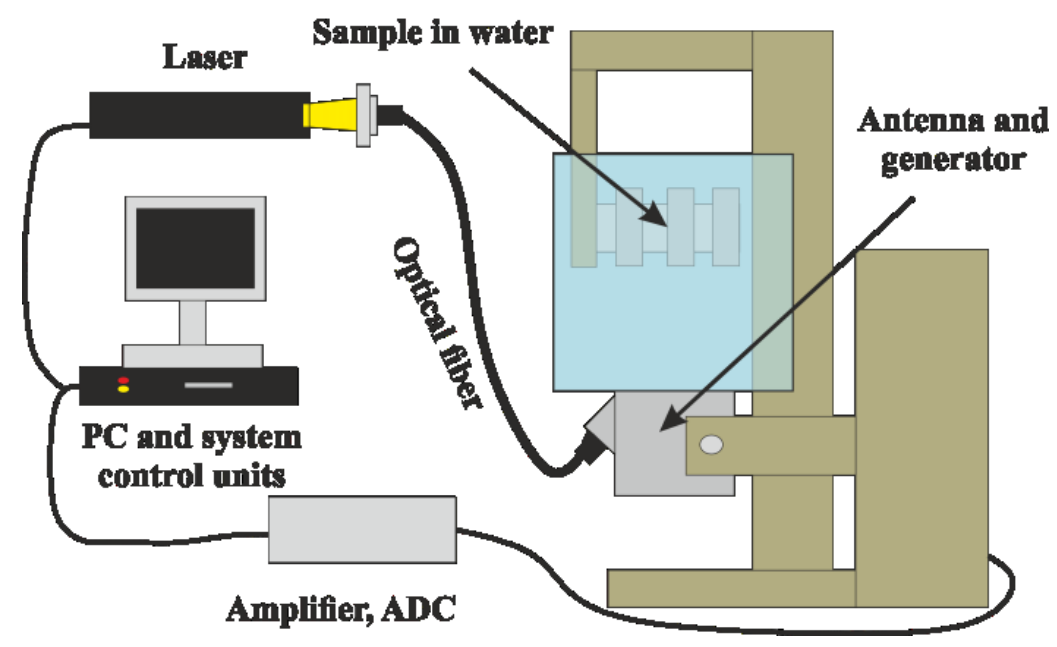

Fig. 1. Schematics of the experimental prototype for laser ultrasound profilometry.

Following this algorithm, the reconstructed acoustical pressure is defined by a formula

$$
\epsilon(r)=\frac{\sum_{i=1}^{N} \Delta \Omega_{i} \times b\left(d_{i}, t=\frac{z+\left|d_{i}-r\right|}{c}\right)}{\sum_{i=1}^{N} \Delta \Omega_{i}}
$$

where $\epsilon(\mathbf{r})$ denotes effective distribution of scatterers (tomogram values) in point with position defined by vector $\mathbf{r}, \mathbf{d}_{\mathrm{i}}$ - position of the $\mathrm{i}^{\text {th }}$ transducer, $N$ - number of transducers, $\Delta \Omega_{i}$ - solid angle of the $\mathrm{i}^{\text {th }}$ transducer as it is seen from point defined by vector $\mathbf{r}$. A solid angle is introduced here to take into account a rate of acoustic energies, received by different transducers. And $b\left(\mathbf{d}_{\mathrm{i}}, t\right)$ is a backprojection value, calculated as

$$
b\left(\mathbf{d}_{\mathrm{i}}, t\right)=-2 \frac{\partial p\left(\mathbf{d}_{\mathrm{i}}, t\right)}{\partial t}
$$

where $p\left(\mathbf{d}_{\mathrm{i}}, t\right)$ - pressure, detected by $\mathrm{i}^{\text {th }}$ transducer.

After the reconstruction of tomograms, the profile of objects has to be detected on them. As it is seen from formula (1), the calculation of tomograms with sufficient number of pixels (typically $800 \times 800$ pixels for image of $20 \mathrm{~mm} \times 20 \mathrm{~mm} \mathrm{size)} \mathrm{is} \mathrm{the} \mathrm{time}$ 
consuming operation even not taking into account profile recognition. Therefore, the algorithms were implemented using parallel programming technology on graphical processing units NVIDIA CUDA [11].

For the spectral filtering of raw signals a bandpass filter was used. This filter is defined by a formula

$$
K(f)=K_{0} \exp \left(-\left(\frac{f_{l}}{f}\right)^{8}-\left(\frac{f_{l}}{f}\right)^{2}-\left(\frac{f}{f_{h}}\right)^{4}\right)
$$

where $f_{l}$ and $f_{h}$ - cut-off lower and higher frequencies respectively, which were set to 0.1 $\mathrm{MHz}$ and $10 \mathrm{MHz}$ in experiments. Both frequencies in (4) are determined by spectral band effectively acquired by PVDF transducers as well as by noise spectrum.

Several bodies of rotation were used as reference samples: a cylinder, a cylinder with irregular grooves and a cylinder with regular grooves. A photograph of the samples is shown in Fig. 2, and the tomograms obtained using the experimental setup are shown in Fig. 4. The main features of the samples are clearly revealed by these tomograms. Therefore, the next step is the implementation of algorithms for automatized profile detection from previously reconstructed tomograms.

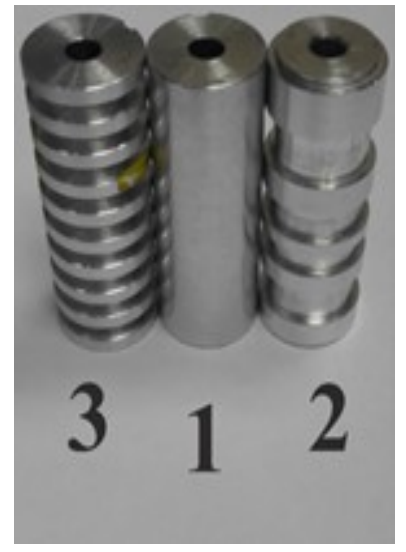

Fig. 2. Reference samples. 1 - cylinder, $2-$ cylinder with irregular grooves, 3 - cylinder with regular grooves.

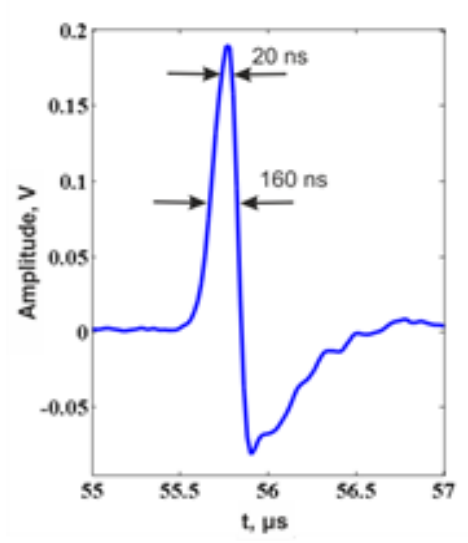

Fig. 3. Scattered from the cylinder surface signal.

A two-step algorithm was proposed for this purpose.

1. Determination of the position of the tomogram peaks in each column (parallel to the vertical z-axis direction, see Fig. 4). Theoretically this operation as follows from NyquistShannon theorem can be carried out with arbitrary precision if the sufficient sampling rate is provided. Within the current experiment the condition of the theorem is fulfilled: the sampling rate of $50 \mathrm{MHz}$ is more than 2 times greater than the significant upper frequency of the signal $(15 \mathrm{MHz})$. The definition of the maximum position was conducted in two stages. Initially, an approximate position of the maximum was determined, after which a parabolic interpolation of the tomogram in a vicinity of the maximum was used to refine maximum position (Fig. 3).

2. After a set of the tomogram maxima is determined, the Hough transform [9] and the least-squares method were used to determine linear sections on the profile, their equations, and line approximation errors. As it follows from the experimental results, the 
approximation inconsistency of the cylinder surface is $15 \mu \mathrm{m}$. This confirms the possibility of the reliable detection of solid surfaces profile with high precision.

\section{Results and discussion}

To verify the results of the profiler prototype measurements, a comparison with a measurements carried out by X-ray tomograph XD7600NT was done for cylindrical samples with regular and irregular grooves.

The equations of the outer and inner surfaces of the regular grooves were obtained

$$
\begin{gathered}
y=k \cdot x+b \\
k_{1}=(-2.24 \pm 0.03) \cdot 10^{-2}, b_{1}=(0.930 \pm 0.002) \mathrm{mm} \\
k_{2}=-2.14 \pm 0.03, b_{2}=(2.902 \pm 0.001) \mathrm{mm}
\end{gathered}
$$

where coefficients errors are standard errors calculated following linear least-squares method.

From equations it follows that the depth of the grooves defined by the prototype is $1.972 \pm 0.005 \mathrm{~mm}$. Measured with an X-ray tomograph, the depth of the grooves is found to be equal to $1.968 \pm 0.005 \mathrm{~mm}$. Therefore, the longitudinal measurements coincide within the instrumental error (Fig. 5).
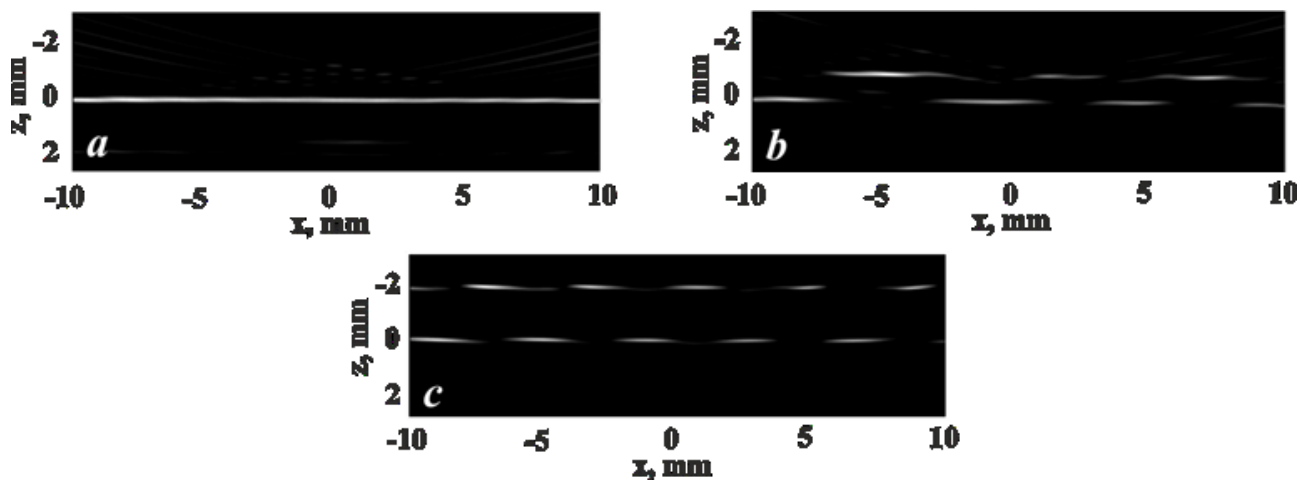

Fig. 4. Tomograms obtained using automatized prototype. a) Cylinder, b) cylinder with irregular grooves, в) cylinder with regular grooves.

The next inspected reference sample is the cylinder with irregular grooves. Measured with the developed laser-ultrasonic profilometer prorotype depth is $0.988 \pm 0.004 \mathrm{~mm}$, while the X-ray tomograph obtained $0.950 \pm 0.005 \mathrm{~mm}$. The discrepancy of the results is due to the fact, that the algorithm for calculation of the straight lines gives an average value of the depth over all grooves, and X-ray tomograph gives a depth measurement of a single groove.

One of the issues related to the profilometer prototype is its low transversal resolution. The combination of a receiving elements large width $(1 \mathrm{~mm})$ and of the small number of transducers leads to the high errors in determination of the positions of the grooves boundaries (up to 100-200 $\mu \mathrm{m}$ ). It's possible to improve prototype transverse resolution in several ways. For instance, an antenna of modified configuration (e.g. toroidal with 
increased number of transducers) could be used. Another way is the development of techniques for reconstruction of composite images obtained from a set of tomograms with a translational or rotational shift.

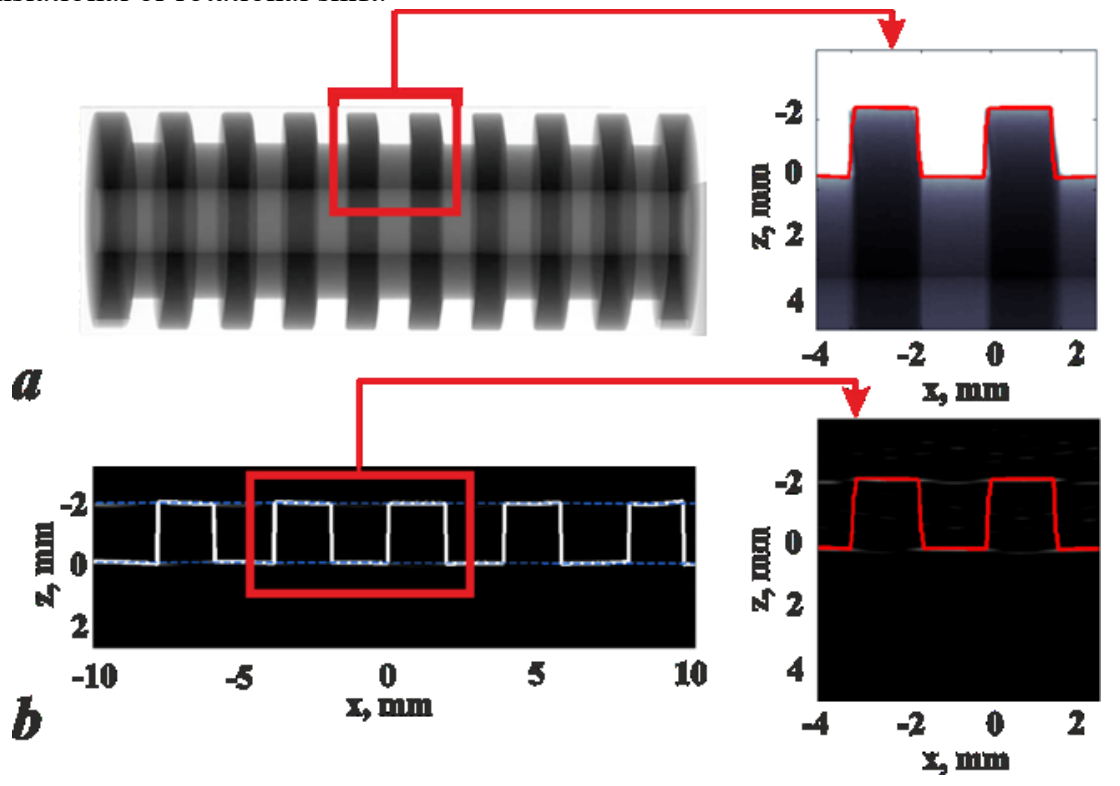

Fig. 5. a) X-ray tomogram of the sample with regular grooves, b) laser ultrasound tomogram of the sample with regular grooves.

The task of laser-ultrasonic profilometry with high precision is one of the important steps on the way to the development of the method of laser-ultrasonic tomography of solid objects with a curved surface. One of the applications of the laser-ultrasonic tomography method could be the flaw detection in specimens with curved surfaces, which could simplify the control of such samples, at the same time maintaining high spatial resolution.

Among the factors affecting the resolution of profilometry, there are several that require a particularly detailed study [12]. Here, the influence of the finite dimensions of the receiving elements is especially important. The integration of the acoustic signal over the surface of the piezoelectric element leads to the possibility to obtain only a locally averaged position of the surface of the object. This effect, leading to the decreased resolution, however, can be used to calculate the local roughness of objects by the increased duration of the ultrasonic pulse caused by its prolongation due to reflection on inhomogeneities.

\section{Conclusions}

Within the framework of the current study, a prototype of a laser-ultrasonic profilometer operating in real time is designed and tested. Filtered back-projection algorithm and the group of algorithms for tomogram processing and profile detection are parallelized using NVIDIA CUDA parallel programming framework and implemented on graphics processors. The best achieved inconsistency of profile approximation is $15 \mu \mathrm{m}$ for a cylindrical surface. The measurements of the depth of the sample grooves performed by the laser-ultrasound prototype and the X-ray tomograph are compared. In general, the results of comparison showed the coincidence of measurements within the instrumentation error of measurements. According to the results of the current investigation, laser ultrasound 
profilometry is a perspective method for high-speed profilometry, which could be used for inspection of bodies immersed in opaque liquids.

This work was carried out with financial support from the Ministry of Education and Science of the Russian Federation in the framework of the NUST MISiS Competitiveness Program (No. K1-2015025), and by the Russian Science Foundation (Grant No. 16-17-10181).

\section{References}

1. L.C. Lynnworth, Ultrasonic measurements for process control: theory, techniques, application (Academic press, 2013).

2. L.T. de Haan, V. Gimenez-Pinto, et. al. Adv. Funct. Mater. 24, 9 (2014).

3. B.H. Lu, J.L. Zhang, B.G. Liu, Y.H. Sun, G.D. Liu, Proc. SPIE, Selected Proceedings of the Photoelectronic Technology Committee Conferences 1, (2014)

4. D.H. Lee, N.G. Cho, Meas. Sci. Technol. 23, 10 (2012)

5. J. Schmit, J. Reed, E. Novak, J.K. Gimzewski, J. Opt. A : Pure Appl. Opt. 10, 6 (2008)

6. M.F. Costa, J. Indian Inst. Sci. 76, 2 (2013)

7. A. Briggs, O. Kolosov, Acoustic microscopy (Oxford University Press, 2009)

8. V.E. Gusev, A.A. Karabutov, Laser Optoacoustics (American Institute of Physics, 1993)

9. V.A. Simonova, E.V. Savateeva, A.A. Karabutov, Moscow Univ. Phys. Bull. 64, 4 (2009)

10. G. Wurzinger G., R. Nuster, N. Schmitner, S. Gratt et al., Biomed. Opt. Express. 4, 8 (2013)

11. R. Szeliski, Computer vision: algorithms and applications (Springer Science \& Business Media, 2010)

12. I.M. Pelivanov, V.A. Simonova, T.D. Khohlova, A.A. Karabutov, Proc. SPIE, Photons Plus Ultrasound: Imaging and Sensing, 756428 (2010) 